\title{
Isolated tubercular hepatic abscess with diffuse pattern mimicking hepatocellular carcinoma in HIV positive patient: a case report
}

\author{
MPHO SETIME ${ }^{1}$, GODFREY M. RWEGERERA ${ }^{2 *}$ and WAHHAB CHOWDHURY ${ }^{3}$ \\ ${ }^{1}$ Department of Internal Medicine, Princess Marina Hospital, Gaborone, Botswana \\ ${ }^{2}$ Department of Internal Medicine, University of Botswana, School of Medicine and Princess Marina Hospital, \\ Private Bag 00712, Gaborone, Botswana \\ ${ }^{3}$ Department of Histopathology, National Health Laboratory, Gaborone, Botswana
}

\begin{abstract}
Isolated hepatic tuberculosis presenting as a mass either with or without fever can be confused with hepatocellular carcinoma. Clinical examination and laboratory investigations are not specific. Radiological investigations such as ultrasound and computed tomography cannot confirm the diagnosis; hence it is vital to always make an effort to demonstrate presence of acid fast bacilli in aspirated pus or necrotic material from a liver mass. We present a case of 50 years old male patient with HIV/AIDS who presented with non-specific symptoms without fever, clinically with hepatomegaly mimicking hepatocellular carcinoma with metastasis. Abdominal ultrasound revealed a mass in the left liver lobe, with diffuse involvement in the right liver lobe. Liver mass histology showed granulomatous pattern with epitheloid cell aggregation. Aspirated blood from fine-needle guided biopsy stained on Ziehl Neelsen for acid fast bacilli turned out positive. Clinical examination did not reveal lymphadenopathy, abdominal ultrasound also ruled out para-aortic lymphadenopathy, and chest $\mathrm{x}$-ray was essentially normal. Evaluation of the patient six months after completing quadruple treatment for tuberculosis showed marked clinical improvement. The objective of this case report is to highlight the importance of considering hepatic tuberculosis as a differential diagnosis in cases of hepatomegaly and initiate appropriate investigations to rule out a possibility of Tuberculosis which is potentially treatable with early diagnosis.
\end{abstract}

Keyword: hepatic abscess, tuberculosis, diffuse pattern, HIV/AIDS, acid fast bacilli, Botswana

\section{Introduction}

Isolated liver tuberculosis (ILT) either in the form of tuberculoma or abscess is still considered a rare condition and hepatic tuberculosis is usually associated with active pulmonary or miliary tuberculosis (Singh et al., 2004). Tubercular liver abscess, when present, is usually associated with the focus of infection in the lung or in the gastrointestinal tract (Javid, 1998), or is associated with an immunocompromised state (Maharaj et al., 1987; Amarapurkar et al., 1995). Cases of isolated hepatic tuberculosis are also not uncommon in immunocompetent patients especially with history of contact with patients with tuberculosis (Hassan et al., 2010; Bhatt et al., 2013). The pathogenesis of hepatic tuberculosis is by haematogenous dissemination, the portal of entry being hepatic artery for hepatic miliary tuberculosis and portal vein for focal liver tuberculosis (Javid et al., 1998). Despite the incidence of tuberculosis increasing with the pandemic of HIV/AIDS in Sub-Saharan, there are few documented cases of isolated hepatic tuberculosis presenting with a focal lesion and diffuse manifestation at the same time.

Abdominal tuberculosis has been associated with significant mortality reaching up to $50 \%$ in previous studies especially when diagnosis is delayed (Alcantara-Payawal et al., 1997; Chen et al., 2003), hence the objective of this case report is to highlight significance of including hepatic tuberculosis in the differential diagnosis of all cases of hepatomegaly and initiate appropriate investigations, with potential of early treatment and improvement in clinical outcomes.

'Correspondence: Godfrey M. Rwegerera; E-mail: godfreyrwegerera@gmail.com 


\section{Case presentation}

A 50 years old male was referred to Princess Marina Hospital, a tertiary hospital in Gaborone, Botswana for further evaluation of a liver mass on 14/05/2013. He gave a history of abdominal pain, poor appetite, weight loss, fevers and general body malaise for more than three months. He had presented to the local hospital three weeks earlier where he was admitted and treated empirically as a case of liver abscess with a course of two weeks antibiotics namely Intravenous Cefotaxime 1 gm 12 hourly, Intravenous Gentamicin 8omg 12 hourly and Tablets Metronidazole 80omg 8 hourly.

It was noted that the patient was afebrile throughout his three weeks admission period at a local hospital. He was referred to a tertiary hospital due to a clinically worsening abdominal pain. There was no history of cough or chest pains. Patient also denied history of being diagnosed or treated for Tuberculosis in the past. He denied previous history of open tuberculosis contact and there was neither history of previous jaundice nor pruritis. He was HIV positive on (Tenofovir, Emtricitabine and Effavirenz) since December 2012, with baseline CD4 92 cells/ $\mu \mathrm{L}$.

Examination revealed a middle-aged man who was cachectic, afebrile, mild pale, no lymphadenopathy or clubbing. . Vitals were stable. Examination revealed a distended abdomen, enlarged, rough, tender right hypochondrium mass extending to the epigastrium. No splenomegaly or ascites or any other masses found. Central nervous system, cardiovascular, respiratory and musculoskeletal examinations were not remarkable. The impression of liver mass with differentials of hepatocellular carcinoma, metastatic liver disease and liver abscess were made. Initial workup included Full blood count (FBC) which showed a white cell count of $10.6 \times 10^{9} \mathrm{cells} / \mathrm{l}$ with normal differentials, Haemoglobin of $7.2 \mathrm{~g} / \mathrm{dl}$ (MCV of $90 \mathrm{fl}$ ) and platelet count of $566 \times 10^{9} / \mathrm{l}$, raised alkaline phosphatise (ALP) of $217 \mu \mathrm{mol} / \mathrm{l}$, hypoalbuminaemia of $23.9 \mathrm{~g} / \mathrm{l}$. The rest of liver enzymes and renal function tests were normal. Hepatitis $B$ and $C$ screening were negative, Alpha-fetoprotein was normal at $0.74 \mathrm{ng} / \mathrm{dl}(0-20)$. Carcinoembryonic antigen (CEA) and Prostatic specific antigens (PSA) were also normal at $<1 \mathrm{ng} / \mathrm{ml}$ and $2 \mathrm{ng} / \mathrm{ml}$ respectively. Serum amoebic titres and indirect haemaglutination test were not performed as they are unavailable at our facility Stool was negative for Entamoeba histolytica. Induced sputum for Acid Fast Bacilli was negative. Chest x-ray was normal and abdominal ultrasound was significant for hepatomegaly with an irregular hypoechoic mass in the left liver lobe. Right liver lobe had small multiple masses and other organs were unremarkable.

Computed Tomography (CT) abdomen revealed heterogeneous liver mass measuring $7.01 \mathrm{~cm}$ AP, $8.50 \mathrm{~cm}$-Transverse, $6.30 \mathrm{~cm}$-Cranial-Caudal in the left liver lobe. Necrotic areas with no enhancement within the mass were noted (Figure 1). There were multiple ill-defined hypodense areas in the right liver lobe peripherally (Figure 2).

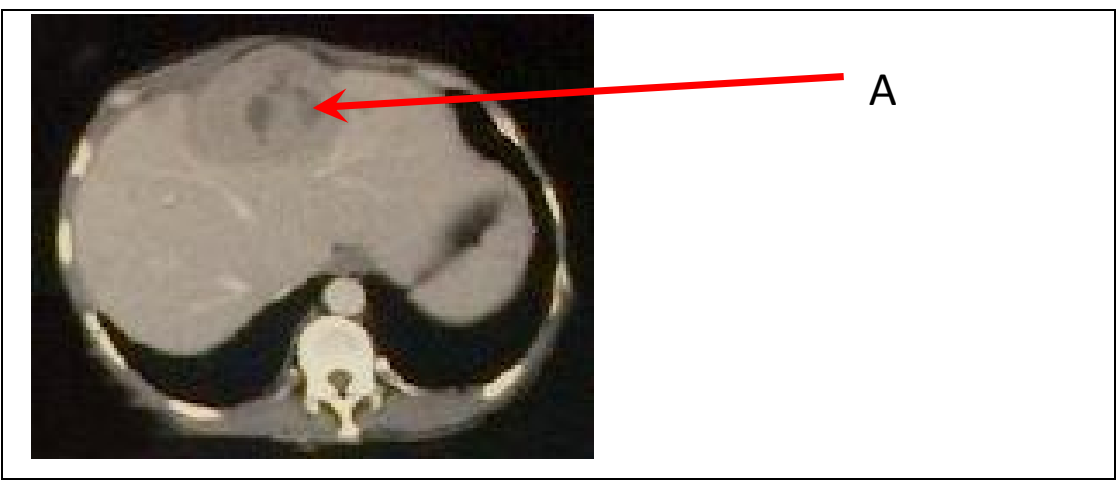

Figure 1: Computed tomography scan of the abdomen showing left liver mass (A) with areas of necrosis 


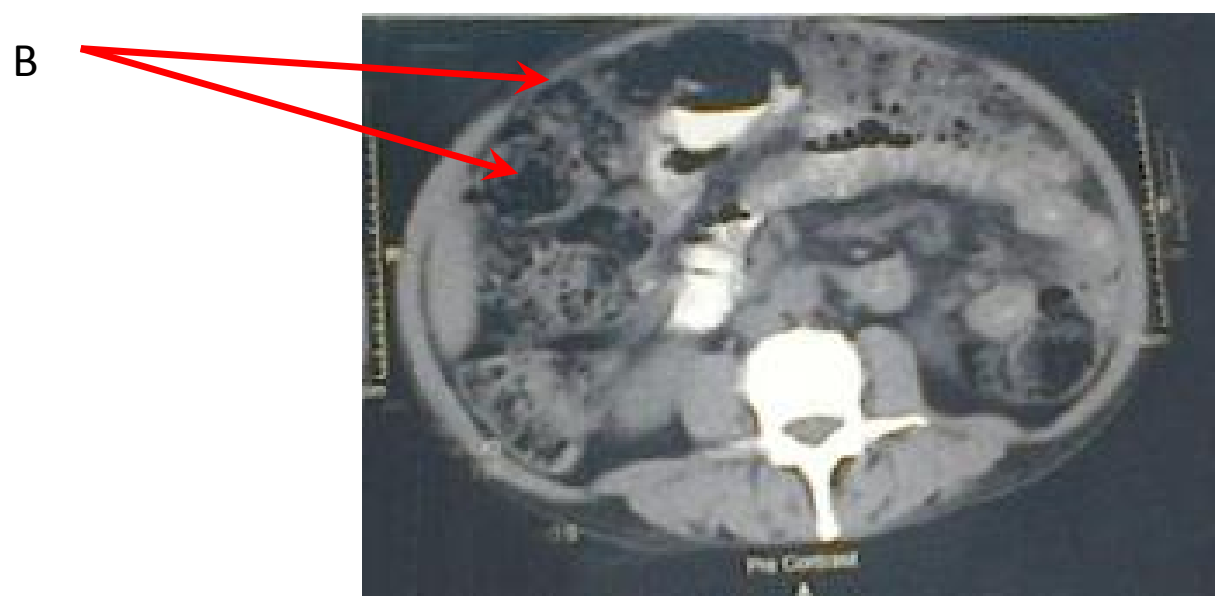

Figure 2: Computed tomography scan of the abdomen showing multiple hypodense ill-defined lesions (B) on the right liver lobe

Ultrasound guided fine needle aspirated blood mixed with pus in a liver mass and showed a neutrophilic rich pattern of inflammatory exudate with presence of aggregates of epitheloid histiocytes, Langeharn's giant cells, macrophages, lymphocytes, fibroblasts and necrotic tissue, consistent with cold abscess probably due to tuberculosis. Culture of aspirated pus did not yield any growth both at 48 and 72 hours. Cell block examination showed pus and granuloma composed of epitheloid cell aggregation. The Ziehl-Neelsen Stain for Acid Fast Bacilli (AFB) was positive. Serum Angiotensin converting enzyme (ACE) to rule out sarcoidosis could not be performed due to lack of the capacity at the facility. Ultimately, the patient was started on quadruple therapy for tuberculosis comprised of isoniazid, rifampicin, pyrazinamide and ethambutol, and monitored at a local clinic. Review of the patient six months after completing antituberculosis treatment revealed a significant clinical improvement, the patient had gained weight, there was no abdominal pain, and physical examination was negative for hepatomegaly.

\section{Discussion}

Experience on previous cases of hepatic tuberculosis has led to three main classifications: diffuse hepatic involvement with pulmonary or miliary tuberculosis accounting for about $50 \%-80 \%$ of cases. Diffuse hepatic infiltration without recognizable pulmonary involvement was the second most common and isolated focal tuberculoma or abscess with an overall incidence of about $0.3 \%$ (Purl et al., 1994; Kok \&Yapp, 1999; Lee et al., 2010). The patient presented in this case was found to have hepatic involvement without evidence of tuberculosis elsewhere; the space occupying lesion in the form of abscess is one of the rarest manifestations. Presence of multiple small hypodense areas in a $\mathrm{CT}$ scan in the right lobe of the liver suggests a diffuse pattern, hence indicating two forms of hepatic tuberculosis coexisting in a patient with HIV/AIDS. The clinical presentation of this patient with abdominal pain, and weight loss in the absence of fever throughout his admission both at local and tertiary hospitals was confusing making hepatocellular carcinoma most likely. Despite the fact that there is no pathognomonic feature of local hepatic tuberculosis, some or all symptoms together were present in most of the previous case reports (Oliva et al., 1990; Hassan et al., 2010; Bhatt et al., 2013).

Our patient was immunosuppressed, his liver enzymes were normal except for alkaline phosphatase which was elevated, this was consistent with other previous cases in 
immunocompetent patients with isolated tuberculosis (Oliva et al., 1990; Achem et al.,1992). The histological picture of hepatic tuberculoma is usually that of a large epitheloid tumour, Langerhantype giant cells with granuloma formation surrounded by lymphohistiocytic cells (Tan et al., 1997). The same picture was also found in our patient and the pus aspiration confirmed the mass to be an abscess. The findings of granuloma formation may suggest possibility of tuberculosis. However, other conditions like primary biliary cirrhosis, sarcoidosis, Crohn's disease, chronic active hepatitis and extra-hepatic biliary obstruction may have the same histological pattern (McCluggage \& Sloan, 1994).

The possibility of other granulomatous diseases was ruled out through history, physical examination and laboratory investigations. Sarcoidosis which also presents with histological pattern of granulomas was ruled out based on absence of skin lesions, clear chest hilar with no evidence of widening mediastinum. In our patient, the diagnosis was reached through bacteriological evaluation with staining aspirate material for Ziehl-Neelsen staining. This highlights the importance of doing this simple, yet forgotten investigation in most of the patients presenting with hepatomegaly. Clinicians should however remember that, the yield of positive acid-fast smears is low (Mandell et al., 1985) and high yield of diagnosis can always be achieved by proceeding to culture of pus or necrotic materials and PCR when available.

In conclusion, hepatic tuberculosis is a potentially treatable condition with satisfactory radiological and clinical outcomes, it calls for high TB-suspicious index among health care workers, especially among HIV/AIDS patients presenting with non-specific manifestations. This will enhance proceeding to appropriate diagnostic investigations including Ziehl- Nieelsen staining, culture of pus or necrotic material or PCR of liver aspirates and prompt antiturbeculosis treatment.

\section{References}

Achem, S.R., Kolts, B.E., Grisnik, J., MacMath, T., Monteiro, C.B. \& Goldstein, J. (1992) Pseudotumoral hepatic tuberculosis. Atypical presentation and comprehensive review of the literature. Journal of Clinical Gastroenterology 14, 72-77.

Alcantara-Payawal, D.E., Matsumura, M. \& Shiratori, Y. (1997) Direct detection of Mycobacterium tuberculosis using polymerase chain reaction assay among patients with hepatic granuloma. Journal of Hepatology 27,620-27.

Amarapurkar, D.N, Chopra, K.B., Phadke, A.Y., Sahni, S., Prabhu, S.R. \& Kairo, R.H. (1995) Tuberculous abscess of the liver associated with HIV infection. Indian Journal of Gastroenterology 14, 21-22.

Bhatt, G.C., Nandan, D. \& Singh, S. (2013) Isolated tuberculous liver abscess in immunocompetent children - report of two cases. Pathogens and Glob Health 107,35-7.

Chen, H.C., Chao, Y.C., Shyu, R.Y. \& Hsieh, T.Y. (2003) Isolated tuberculous liver abscesses with multiple hyperechoic masses on ultrasound: a case report and review of the literature. Liver International 23,346-50.

Javid, G., Khan, B., Shah, A. \& Khan, M.A. (1998) Isolated tuberculous liver abscess. The Journal of the Association of Indian Physician 46,973-974.

Hassan, K.M., Ousadden, A., Ankouz, A., Mazaz, K. \& Taleb, K.A. (2010) Isolated liver tuberculosis abscess in a patient without immunodeficiency: A case report. World Journal of Hepatology 2,354-357.

Kok, K.Y. \& Yapp, S.K. (1999) Isolated hepatic tuberculosis: report of five cases and review of the literature. Journal of Hepato-Biliary-Pancreatic Surgery 6,195-198.

Lee, S.W.,Lien,H.C. \& Chang,C.S (2010) Tuberculous liver abscess in a case without lung involvement. Kaohsiung Journal of Medical Sciences 26, 99-104. 
Maharaj, B., Leary, W. \& Pudifin, D. (1987) A prospective study of hepatic tuberculosis in 41 black patients. Quarterly Journal of Medicine 63,517-522.

Mandell, G.L., Gordon-Douglas, R. Jr., Bennett, J.E. (1985) Principles and Practice of Infectious Diseases, 2nd edition. New York: John Wiley \& Sons, 791-794pp.

McCluggage, W.G., Sloan, J.M. (1994) Hepatic granulomas in Northern Ireland: a thirteen year review. Histopathology 25, 219-228.

Oliva, A., Duarte, B., Jonasson, O. \& Nadimpalli, V. (1990) The nodular form of local hepatic tuberculosis. A review. Journal of Clinical Gastroenterology 12,166-73

Purl, A.S., Nayyar, A.K. \& Vij, J.C. (1994) Hepatic tuberculosis. Indian Journal of Tuberculosis 41, 131-134

Singh, D., Singh, S., Raut, S. \& Karmarkar, S. (2004) Isolated liver tuberculosis: a case report. Pediatric Surgery International 20, 727-728.

Tan, T.C., Cheung, A.Y., Wan, W.Y. \& Chen, T.C. (1997) Tuberculoma of the liver presenting as a hyperechoic mass on ultrasound. British Journal of Radiology 70, 1293-1295. 